\title{
Treatment of Kidney Stone Disease, Benign Prostatic Hyperplasia and Chronic Prostatitis with ThermobalancingTherapy should be listed in the Guidance for Health Professionals
}

\author{
Simon Allen., MD, PhD, Academician* \\ Director Fine Treatment, Oxford, United Kingdom
}

*Corresponding Author: Simon Allen, MD, PhD, Academician, Director Fine Treatment, Oxford, United Kingdom. Email: info@finetreatment.com

\begin{abstract}
The prevalence of kidney stone disease, chronic prostatitis / chronic pelvic pain syndrome $(C P / C P P S)$ and benign prostatic hyperplasia (BPH) increases around the world. Long-term medications and surgical procedures for these non-life-threatening chronic conditions can develop serious side effects and complications. At the same time, Thermobalancing therapy (TT) with Dr Allen's therapeutic Device (DATD) has shown its high effectiveness in the treatment of chronic internal diseases. Discussion at many international conferences in 2017-2018 was aimed at treating various chronic non-malignant diseases with the help of Thermobalancing therapy, such as BPH, CP/CPPS, kidney stones and cardiovascular diseases.

In the conferences were presented cases of TT with DATD of patients with kidney stone disease that confirm the ability of DATD to dissolve any type and size of kidney stone; discussed the outcomes from the clinical trials of TT in 124 men with BPH and 45 patients with CP/CPPS, before and after 6-month period they used DATD. The assured results of the cases and clinical trials reveal that TT with DATD treats the cause of kidney stone disease, CP/CPPS and BPH. Implementation of this therapy is needed today, as it helps patients avoid long-term drugs and surgical procedures, including extracorporeal shock wave lithotripsy (ESWL) with their risks and complications. In addition, it is a cost-effective solution. Therefore, conference participants proposed that TT with DATD should be included in the Guidance for health professionals.
\end{abstract}

Keywords: Enlarged Prostate, Kidney Stones, Prostatitis, Thermobalancing Therapy

\section{BACKGROUND}

Thermobalancing therapy (TT) enabled by Dr Allen's therapeutic device (DATD) is able to treat chronic internal disease. 2 clinical studies on TT in men with benign prostatic hyperplasia $(\mathrm{BPH})$ and chronic prostatitis/chronic pelvic pain syndrome (CP/CPPS) were completed. They confirmed the high effectiveness of this safe therapy that has no adverse side effects $[1,2]$. There ismany empirical evidence that DADT dissolves any type of kidney stones and prevents their recurrence. TT with DATDis effective because it eliminates the constant trigger in the tissue of affected organ, namely the focus of hypothermia, maintaining the source of energy obtained from the body in its projection, stopping the pressure inside the organ, which leads to relief of pain and other symptoms[3]. Discussions at the International Conferences in Spain, China, Italy, London, Paris and other countries (2017-2018) were aimed at better understanding the origin of disease of chronic internal diseases and their treatment.

\subsection{Kidney Stone Disease}

Kidney stone disease or nephrolithiasis is a highly prevalent disease worldwide with rates ranging from 7 to 13\% in North America, 5-9\% in Europe, and 1-5\% in Asia [4]. This disease is also prevalent in mainland China, and the prevalence increases with age. Due to high rates of new and recurrent stones, management of stones is expensive and the disease has a high level of acute and chronic morbidity. There has been a rising incidence in stone disease throughout the world with a narrowing of the gender gap [5,6]

Most patients with ureteral stones present with typical symptoms, although many stones in the renal calices remain asymptomatic. Not every asymptomatic caliceal stone needs to be treated immediately [7]. Ureteral stones $<6 \mathrm{~mm}$ can pass spontaneously in controlled patients, while medical expulsive therapy, without prior 
extracorporeal shock wave lithotripsy (ESWL), supports the chance of successful passage and reduces the need for analgesics [8].

Nowadays, the competition for priority in the treatment of kidney stones between ESWL, percutaneous nephrolithotomy (PCNL) and retrograde intrarenal surgery (RIRs) turned on [9]. After ESWL was implemented it was supposed that $85 \%$ of all patients with kidneys stones could be cured by nonsurgical means [10]. The initial promising results of ESWL were challenge with common side effects caused by this procedure, such as bleeding, severe pain, or urinary tract infections [9], rare complications, such as spleen rupture or intrarenal hematoma $[11,12,13]$, and long-term medical effects: hypertension and diabetes mellitus $[14,15,16]$.

A prospective-randomized study with asymptomatic caliceal stones $<15 \mathrm{~mm}$ did not find any statistical advantage of ESWL over observation in terms of stone free rate (SFR), quality-of-life, kidney function and symptoms during a 2.2-year follow-up [17]. Therefore, watchful waiting can be suggested with yearly follow-up examinations over a period of 2-3 years.Asymptomatic calyceal stones may be observed but there is a higher risk of symptoms and a need for intervention within 5 yr. $[18,19]$.

The cause of pain in kidney stone disease is understood for thousands of years. Kidney stones can cause pain as they pass through ureter, stretching the proximal ureter and renal collecting system. This physical stretch activates nociceptive nerve fibers within an entire neuronal network located submucosally in the renal pelvis, calyces, capsule and ureter [20].

Kidney stones can occur due to excess of elements in blood, infection, dietary changes and other factors. Nevertheless, the pathophysiology of kidney stones is still unknown, and a more complete understanding of stone formation is needed to develop prevention strategies [21]. Only after it was shown that the origin of diseases in all chronic internal diseases has the same root, the formation of kidney stones can be explained as a chain of changes at the microvascular level, and it is supported by evidence that TT with DATDdissolves kidney stones [22].

\subsection{Benign Prostatic Hyperplasia}

Lower urinary tract symptoms (LUTS) secondary to benign prostatic hyperplasia (BPH) most often encountered in men over the age of 50 [23]. Many men with CP/CPPS and BPH are not satisfied with conventional treatment options and they try alternative therapies:diet and lifestyle modifications, phytotherapy and acupuncture [24]. Thus, in the last decade, the necessity of medical/surgical treatment of $\mathrm{BPH}$ has been challenged. BPH/LUTS should not be viewed as an inevitable disease of older people but as part of the aging process that can be prevented [25].

It was shown that acupuncture can relieve the clinical symptoms of prostate hyperplasia and raise the patients' quality of life [26]. The following investigations also demonstrate that the effect of electroacupuncture (EA) on the urinary symptoms was positive but it doesn't improve clinical parameters in patients with moderate to severe BPH [27]. Statistically significant changes were observed in short-term follow-up endpoints in favor of acupuncture in moderate to severe BPH. However, it is not certain whether clinically significant outcomes can be achieved by acupuncture [28].

Phytotherapy doesn't significantly improves symptoms of enlarged prostate against placebo, despite being largely safe for ingestion. However, it can be used in patients with mild $\mathrm{BPH}$ symptoms who are reluctant to take standard pharmaceutical medications provided that men understand their current limitations [29]. Therefore, recommendations on the evaluation and treatment of LUTS in older men are in demand.BPH treatment should be holistic, and may include conservative measures, lifestyle interventions and behavioral modifications, as well as medication and surgery [30].

Many researchers have tried to understand the etiology and pathophysiology of BPH in order to find the optimal treatment for this frequent disease, but they concluded that the exact cause of BPH remains largely unknown [31,32].

TT with DATD by using continuous heat exposure that does not exceed the normal body temperature terminates the trigger of $\mathrm{BPH}$ development, the micro-focus of hypothermia, and the following spontaneous expansion of capillaries; stops and even reverses prostate enlargement. Thus, it was confirmed that the 
$\mathrm{BPH}$ cause related to the chain of changes at the microvascular level [33].

\subsection{Chronic Prostatitis/Chronic Pelvic Pain Syndrome}

$\mathrm{CP} / \mathrm{CPPS}$ is a common condition that causes severe symptoms and worsens quality of life (QoL) in $8.2 \%$ of affected men [34].Chronic pain, the main symptom of CP/CPPS, which is characterized by pelvic pain and voiding symptoms, is poorly defined [35]. Pelvic pain may have a close relationship with the pelvic floor, as it has been established potential therapeutic benefit from pelvic floor therapy for men with CP/CPPS [36]. However, chronic pain is a multifactorial condition, caused by the complex interplay of nociceptive, neuropathic or mixed pathogenic mechanisms [37]. It maybe because of pain CP/CPPS also takes a substantial toll on physical and mental health, and remains a major challenge and is a source of frustration to both the patient and physician since standard treatments often fail and a "panacea" is still lacking[38].

Different etiologies and mechanisms of CP/CPPS pathogenesis have been proposed with a suggested immunological, neurological, endocrine, or psychological basis [39]. For instance,hypogonadism, a treatment of which is raise in the aging population [40] with the appropriate use of testosterone replacement therapy [41], today may be suggested as target in men with CP/CPPS [42].However, as there is no successful treatment for this common health problem, a treatment methodology for CP/CPPS is needed [43].

TT with DATD by providing continuous application with a natural source of energy to the prostate gland reaches a marked improvement of quality of life (QoL) and symptomatic relief in men with CP/CPPS [44]. The initiating factors for CP/CPPS may differ but the trigger for the development of chronic disease is the focus of hypothermia and, consequently,ischemia, sets up a vicious cycleof spontaneousgrowthof capillaries and continuous pressure inside the prostate tissue that leads to CP/CPPS.DATD terminates the focus of hypothermia improves microcirculation in the prostate, affecting the etiology of this disease [45].

\subsection{The Purpose of the Research Review}

The purpose of this review is to find an optimal treatment option for people with chronic internal diseases, such as kidney stones disease, BPH and CP/CPPS. Clinical empirical evidence and trials have confirmed that the use of DATD is incredible effective for patients with these chronic conditions.TT with DATD received a patent in the US. The patent is an acknowledgement that it is indeed a completely new treatment option for chronic internal diseases. This review includes discussions at international conferences in Spain, China, Italy, the United Kingdom and France (2017-2018) aimed at a better understanding of the origin of kidney stone disease, BPH, CP/CPPS and recommendations for optimal treatment of these сркщтшс conditions.

\section{MeThODS}

Dr Simon Allen in his speeches at the conferences explained the essence of TT and demonstrated supporting data of its effectiveness. Therefore, below are presented the case studies in patients with kidney stone disease and the outcomes of clinical trials on TT with DATD in men with BPH and CP/CPPS.

\subsection{Study Protocol}

Dr Allen's Device was registered with the Medicines and Healthcare Products Regulatory Agency (MHRA) in the UK in 2010, as a class 1 medical device. A class 1 medical device without a measuring function and supplied in non-sterile condition does not require the involvement of a notified body. So, it is permitted to be used by everyone at home. Therefore, Dr Allen has opened Fine Treatment Company in the United Kingdom that distributes Dr Allen's Device worldwide.

Today we have satisfied customers from over 100 countries. Ethics committee of the Yerevan State Medical University approved the clinical studies on TT with DATD. The studies on BPH and CP/CPPS were registered at the World Health Organisation via the German Clinical Trials Register (DRKS).

\subsection{Study Design}

TT with DATD was used in the clinical controlled studies at The Department of Urology in Yerevan State Medical University. 124 men with $\mathrm{BPH}$ and 45 men with CP/CPPS received TT during 6-month period and their clinical parameters before and after therapy were examined. This information was compared with the control groups, i.e. data received from 124 men with BPH who were in watchful waiting 
and 45 men with CP/CPPS who did not receive TT with DATD. Also, data of patients with kidney stones is presented in this study.

\subsection{The Baseline Evaluations}

It included complete physical examination, medical history, DRE, serum biochemistry, and PSA measurements, electrolytes, urine and renal function tests. Evaluations were made at baseline and 6 months after. The International Prostate Symptom Scale (I-PSS) assessed the dynamics of the BPH patients' conditions; and the condition of 45 men was assessed by measurement of the National Institute of Health chronic prostatitis Symptom index (NIH-CPSI). Prostate volume (PV $\mathrm{mL}$ ) was measured by ultrasonography. The standard ellipsoid formula length $\times$ width $\times$ height $\times 0.52$ was used to determine prostate volume. Dynamics of the symptoms and the indicators in each group were evaluated in comparison to their data in the beginning and end of the treatment.

\subsection{Statistical Analyses}

The independent-samples t-test and pairedsamples t-test are suitable only for interval and ratio data, so the Wilcoxon signed-rank test was employed. $\mathrm{P}<0.05$ was considered significant. Statistical analyses were carried out using SPSS v22 (IBM, Armonk, NY, USA).

\subsection{TT and DATD}

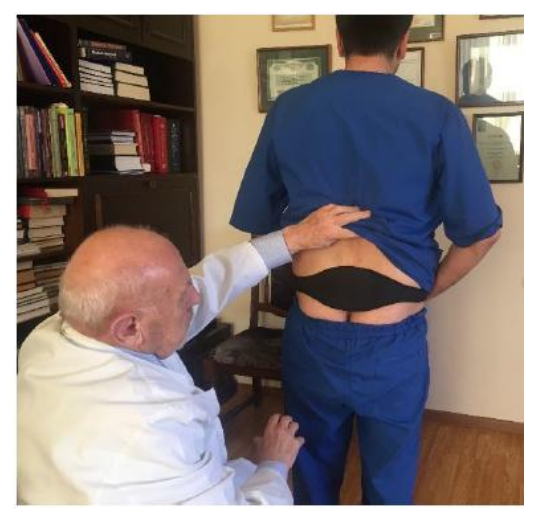

Picture1. DATD tightly attaches thermoelement to the coccyx area for a prolonged period of time.

TT enabled by DATD for the first time in the world treats the affected organ using own energy of the human body. Natural thermoelement(s) in the device accumulates the body heat and becomes a source of energy itself. Thus, it treats a prostate gland by keeping the source of energy, which has been accumulated from the body, in the projection of the prostate during the prolonged period of time, for 6 months and longer, see Picture 1.
The samehappens with patients who suffer with kidney stone disease, when the device applies thermoelements to both kidneys it dissolves stones by keeping the source of energy, which has been accumulated from the body, in the projection of both kidneys during the prolonged period of time, see Picture 2.

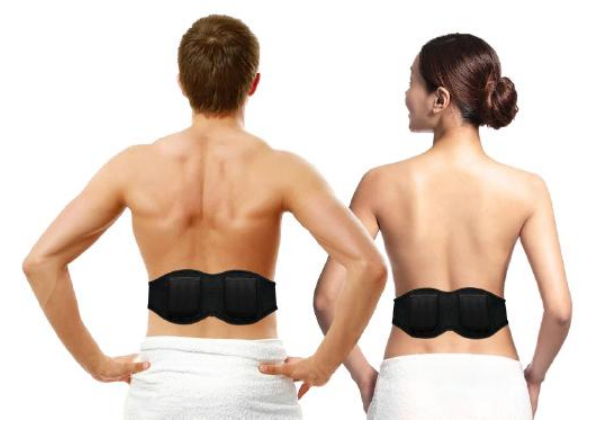

Picture2. Dr Allen's Device for kidney treatment consists of 2 thermoelements and a belt that applies these thermoelements to the projection of both kidneys.

\section{Results}

Table1. Data in this table demonstrates that DATD is effective tool for kidney stone disease. DATD can be used in men and women.

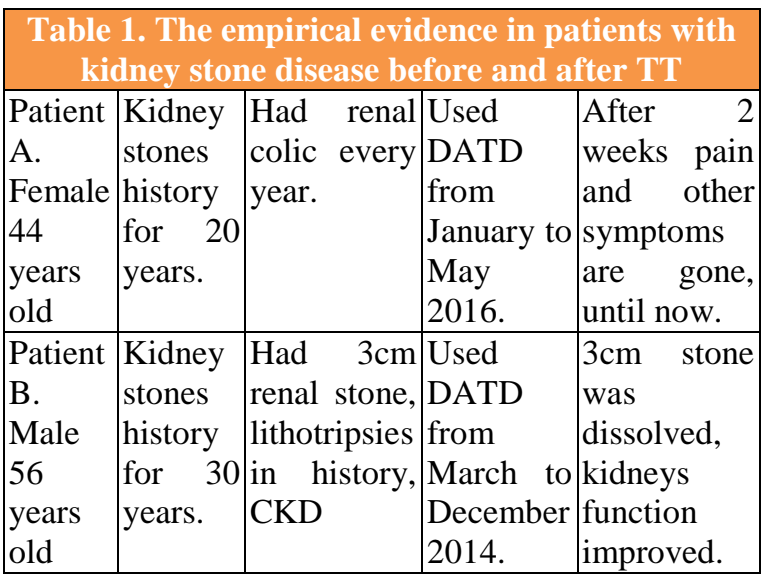

We have collected many cases provided to me by the users and should note that not a single user of Dr Allen's Device for kidney treatment has complained that this treatment method has not worked for him or her. Table 1. presents data from 2 patients with kidney disease who have suffered from this disease for many years and dissolved kidney stones using TT with DATD.

The outcomes of the clinical trial on TT in 124 men with BPH are presented in Figure 1 and Figure 2.

In the control group, the mean IPSS - UrS score increased from $13.45 \pm 3.254$ to $14.35 \pm 3.396$ $(P<0.001)$ whereas, in the treatment group, it 
Treatment of Kidney Stone Disease, Benign Prostatic Hyperplasia and Chronic Prostatitis with Thermobalancing Therapy should be listed in the Guidance for Health Professionals

decreased from $14.33 \pm 3.399$ to $4.73 \pm 2.754$ $(P<0.001)$ at the end of the observation period Hence, DATD decreased urinary symptoms significantly whereas, in the absence of treatment, urinary symptoms worsened significantly.

In the control group, the mean IPSS-QoL score increased from $3.43 \pm 0.956$ to $3.76 \pm 0.983$ $(P<0.001)$ whereas, in the treatment group, it decreased from $3.91 \pm 0.755$ to $1.39 \pm 1.110$ $(P<0.001)$. These results suggested that DATD treatment improved QoL whereas, in the control group, QoL worsened.

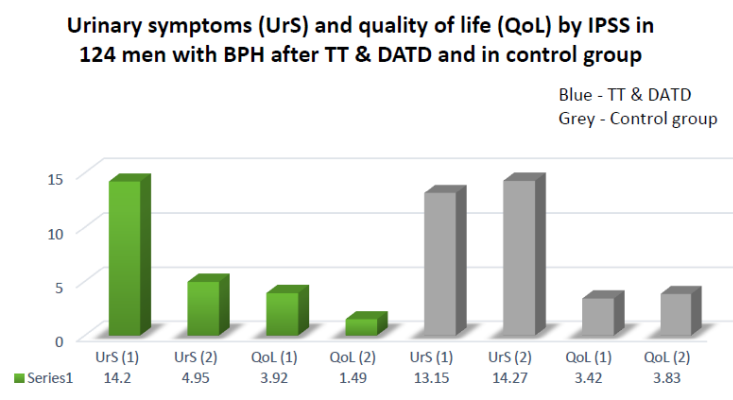

Figure1.Dynamics of the IPSS for urinary symptoms (UrS) and QoL in 124 patients with BPH afterTT with DATD, and in the control group, at the beginning and at end of the study demonstrated:

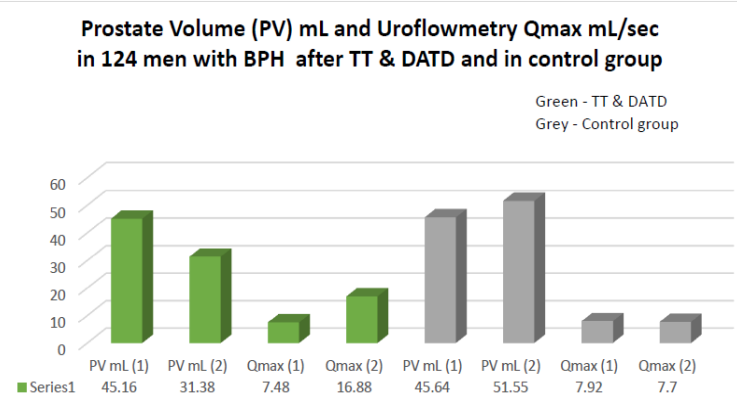

Figure2. Changes in prostate volume $(P V, m L)$ and uroflowmetry, $Q_{\max }, \mathrm{mL} / \mathrm{s}$ ) in 124 men with $B P H$ afterTT with DATDand in the control group, at the beginning and at end of the study demonstrated

In the control group, the mean PV increased from $45.54 \mathrm{~mL}$ to $50.85 \mathrm{~mL}(P<0.001)$ whereas, in the treatment group, it decreased from 45.19 $\mathrm{mL}$ to $31.86 \mathrm{~mL} \quad(P<0.001)$.Hence, DATD reduced PV significantly whereas, in the control group, PV increased.

In the control group, the mean $\mathrm{Q}_{\max }$ decreased from $7.95 \pm 2.871$ to $7.7 \pm 2.695 \mathrm{~mL} / \mathrm{s}$ (no significant difference) whereas, in the treatment group, the mean $\mathrm{Q}_{\max }$ increased from $8.10 \pm 3.041$ to $17.73 \pm 4.392 \mathrm{~mL} / \mathrm{s} \quad(P<0.001)$. These results suggested that DATD increased $\mathrm{Q}_{\max }$ significantly whereas, in the control-group, a significant difference in $\mathrm{Q}_{\max }$ was not observed.

The outcomes of the clinical trial on TT in 45 men with CP/CPPS are presented in Figure 3. and Figure 4.

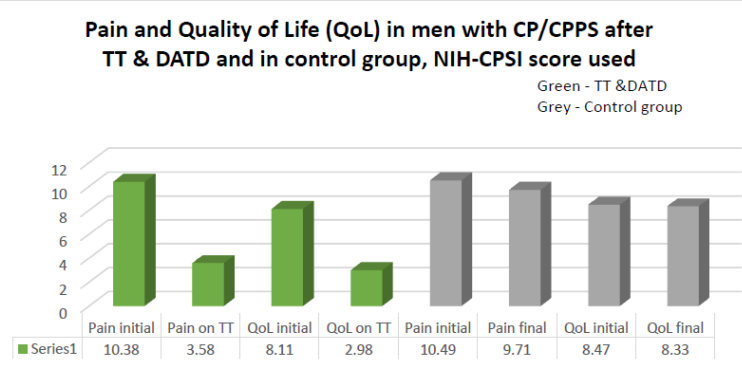

Figure3. Dynamics in Pain and Quality of life evaluation scores in 45 men with CP/CPPS according to the NIH-CPSI before and after TT with DATD and in the control group demonstrated

In the control group, the mean pain score decreased from 10.49 to $9.71(P<0.05)$ at the end of the study period, whereas in the treatment group the mean pain score decreased from 10.38 to $3.58 \quad(P<0.001)$. These data suggest that although the pain score decreased in both groups, the difference between scores was considerably higher $(8.72: 1)$ in the treatment group than the control group.

In the control group Estimation of quality of life showed a decrease from 8.47 to 8.33 (no significant difference), whereas in the treatment group the mean quality of life went from 8.11 to $2.98 \quad(P<0.001)$. We concluded that the treatment improved the quality of life significantly, whereas in the control group there was no significant difference.

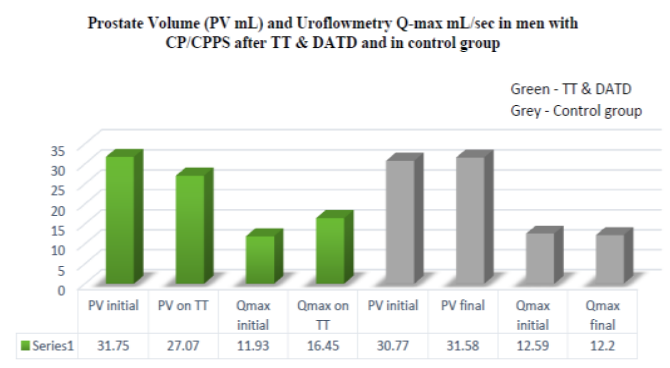

Figure4.Changes inultrasound measurement of prostate volume $(P V \mathrm{~mL})$ anduroflowmetry $Q_{\max }$ $\mathrm{mL} / \mathrm{sec}$ in 45 men with CP/CPPSbefore and after TT with DATD and in the control group demonstrated

In the control group, the mean prostate volume increased from 30.77 to $31.58(<0.05)$, whereas in the treatment group the mean prostate volume decreased from 31.75 to $27.07(P<0.001)$. This indicated that the treatment reduced the PV significantly, whereas in the control group the prostate volume increased over time. 
In the control group $\mathrm{Q}_{\max }$ value decreased from 12.59 to 12.20 (no significant difference), whereas in the treatment group the mean uroflowmetryQ $Q_{\max }$ increased from 11.93 to $16.45(P<0.001)$. Thus, the treatment had a significant increase in the uroflowmetry $Q_{\max }$, while the control group had no change in its value overtime.

\section{DISCUSSIONS}

The study highlights the effectiveness of TT with DATD in the treatment of chronic internal diseases, as it dissolves kidney stones, reduces pain and improves QoL in patients with $\mathrm{CP} / \mathrm{CPPS}$; reduces symptoms of urination and improves QoL in men with BPH and reduces $\mathrm{PV}$ and increases Qmax in both treatment groups with CP/CPPS and BPH.

This study was not randomized. The presence of a "placebo" or "sham" group as a control could provide more statistical rigor with respect to results. However, most men with CP/CPPS have psychological problems, they largely do not respond to medical interventions, and pain syndrome is associated with a decrease in $\mathrm{QoL}$ [46,47]. Similar problems are observed in men with BPH suffering from depression and anxiety, their health-related QoL is considered poor, and their psychological well-being is seriously affected [48,49]. A 6-month period may be considered as a short period of time for taking tablets / placebo, but not for using something attached to the body. Therefore, suggesting that men with CP/CPPS and BPH should wear a "placebo-belt" for 6 months, which does not alleviate the symptoms, it would be very difficult. Typically, patients with $\mathrm{CP} / \mathrm{CPPS}$ and BPH felt relief of symptoms for several weeks after wearing DATD and used the device as needed.

This effectiveness of DATD can be explained in the following way: that TT treats the underlying cause of these chronic diseases, tackles the origin of disease.

\subsection{The Origin of Diseases, Including Kidney Stones, BPH, CP/CPPSand TT with DATD}

The vascular factor plays a decisive role in the pathophysiology and etiology of chronic diseases, so all chronic internal diseases have the same root, namely, a pathological activity of capillaries. Two physiological properties of small blood vessels, named by physiologists: constriction of capillaries and spontaneous expansion of capillaries are responsible for the pathological activity of capillaries. First is the constriction of capillaries in response to different triggers, such as infection, cold, excess of elements in blood, stress and others. It creates the focus of hypothermia in the affected tissue, which gradually becomes a trigger itself, irritating the affected tissue [50].

To improve blood circulation in this affected tissue, the second factor, namely spontaneous expansion of a capillary net occurs [51]. Slowly the expanded capillaries form extra tissue that creates pressure in this area of the organ leading to its malfunction. Thus, the conclusion is that this physical factor is responsible for the development of chronic internal disease. In order to eliminate the focus of hypothermia and, consequently, the pressure inside the organ, and ultimately, its malfunction TT enabled by DATD have been developed [52].

\subsection{Management of Kidney Stone Disease}

Kidney stones may lead to chronic kidney disease (CKD) and stone formers are at increased risk for end-stage renal disease (ESRD) [53,54]. However, the management of kidney stones should be evaluated according to severity of side effects and complications after treatments. This conclusion has been created slowly while researches have understood that the stoneremoving procedure may itself negatively impact the functional integrity of the targeted kidney and perhaps its mate [55].

That is why today researches try to find a connection between dietary factors and the risk of incidence of kidney stones, as they see that lifestyle and dietary factors seem to be increasingly important in prevention of stone disease [56,57]. As the incidence of kidney stones is common in the United States and treatments for them are very costly, researches advise stone removal using surgery or ESWL, and aftermedical and dietary treatments to prevent recurrence of stone [58].However, there is no point in recommending dietary management of kidney stones, as it cannot stop the formation of kidney stones without offering useful alternatives to surgical interventions.

Therefore, the introduction of TT with DATD as a first line treatment in the Guidelines for Nephrology and General Practitioners should be 
carried out as soon as possible, as it stops the formation of kidney stones, extremely safely and economically.

\subsection{Management of Benign Prostatic Hyperplasia}

Even though BPH now is considered a consequence of aging, treatment of this disease centered on medical/surgical intervention to counteract LUTS. Medicaltreatment ofBPHis not efficacious as $52.8 \%$ of men with BPHwere dissatisfied withthe results ofmedical treatmentconductedaccording tocurrent international guidelinesforBPH [59].BPH medications have serious side effects, especially in the long-term[60]. Surgical treatments of prostate were found to be unsafe for nonCaucasian race, so for them conservative treatment might be a reasonable alternative [61]. Additionally, the results of a survey of sexually active men after three different laser surgeries from 2005 to 2010, concluded that all these surgical techniques can have a negative impact on sexual function, and patients with normal preoperative sexuality are more at risk [62].

\begin{abstract}
Although many of medical/surgical interventions were advised to men with LUTS, it was generally done in a non-standardised and unsystematic way. A self-management programme could significantly reduce the frequency of escalation through the treatment cascade and reduce urinary symptoms (as effective as medication), suggesting that selfmanagement could be considered as first-line treatment for men with LUTS [63].
\end{abstract}

The self-management of LUTS due to BPH with the DATD demonstrated its high efficacy and, therefore, therapeutic device should be used before any long-term pharmacotherapy is initiated [64]. Therefore, implementation TT with DATD as the first-line BPH treatment into the Guideline for Urologists and General Practitioners should be carried out as soon as possible.

\subsection{Management of Chronic Prostatitis/ Chronic Pelvic Pain Syndrome, CP/CPPS}

The results of the systematic review and metaanalysis confirmed the current inability to effectively manage CP/CPPS, suggesting the identification of the etiology and role of therapeutic intervention in CP/CPPS in the first place [65]. The lack of effective therapy for CP/CPPS forces health professionals to implement different treatment strategies. For example, illness-focused coping as an important biopsychosocial goal in the management of $\mathrm{CP} / \mathrm{CPPS}$ or transrectal triple physiotherapy in combination with pharmacotherapy in patients with CP/CPPS $[66,67]$.

Rees with collegesin the Guidelines recommend that multimodal/combined therapy should be uniquely designed for each individual patient, adding to first-line antibiotic therapy: a-blocker and/or an NSAID; and an agent targeting neuropathic pain (e.g. pregabalin); and phytotherapy, which, has a modest beneficial effect on symptom improvement in CP/CPPS, and may be considered as a treatment option in treatment-refractory patients [68].Several studies of acupuncture have provided positive results as well as transcutaneous electrical nerve stimulation comparing with traditional treatments for CP/CPPS [69,70]. Acupuncture, compared with standard treatment, it is more effective, so it can also serve as a standard treatment option when it is available [71].However, the duration of the effect after acupuncture is not clear.

Today, with invention of TT with DATD, the quality of life of men with chronic pelvic pain should be changed positively. The vascular changes with the focus of hypothermia in affected prostate tissue play the main role in the cause the pain in CP/CPPS. TT eliminates this constant trigger that backs chronic process. By maintaining a source of energy that does not exceed body temperature in the projection ofa prostate, DATDreduces the pressure inside its tissue, relieving pressure on stress nociceptors, which results in relief of chronic pelvic pain. Therefore, TT with DATD can be recommended as an independent treatment option for patients with CP/CPPS [72].

\section{CONCLUSION}

TT with DATD is an effective tool for the treatment of kidney stone disease, $\mathrm{BPH}$ and chronic prostatitis. Kidney stone disease ought to be treated therapeutically by Thermobalancing therapy in the first instance, helping patients to dissolve kidney stones and to avoid surgical procedures, including lithotripsy, with their risks and complications. The treatment of LUTS due to BPH should start with DATD, as TT can stop and reverse prostate enlargement. TT with DATD provides pain and other symptoms relief in men withCP/CPPS.TT 
with DATD is safeand cost-effective Therefore, it is important to include TT with DATD in the Guidance for health professionals for the treatment of kidney stones, BPH and CP/CPPS.

\section{ACKNOWLEDGMENTS}

I am grateful to the organisers and management team of the International Conferences thatgive opportunity to meet medical professionals from different parts of the world and discuss with them the necessity of making changes in treatment of patients with non-malignant chronic conditions.

\section{REFERENCES}

[1] Allen S, Aghajanyan IG. Benign Prostatic Hyperplasia Treatment with New Physiotherapeutic Device, Urol J, 2015 Nov 14;12(5):2371-2376.

[2] Allen S, Aghajanyan I. Effect of thermobalancing therapy on chronic prostatitis and chronic pelvic pain syndrome, Journal of Clinical Urology, 2016, Sept 20, 1-8, doi: 10.1177/2051415816671036.

[3] Allen, S. The Vascular Factor Plays the Main Role in the Cause of Pain in Men with Chronic Prostatitis and Chronic Pelvic Pain Syndrome: The Results of Clinical Trial on Thermobalancing Therapy.Diseases 2017, 5(4), 25; doi:10.3390/diseases5040025.

[4] Sorokin I, Mamoulakis C, Miyazawa K, Rodgers A, Talati J, Lotan Y. Epidemiology of stone disease across the world, World $\mathbf{J}$ Urol. 2017 Sep;35(9):1301-1320. doi: 10.1007/s00345-017-2008-6.

[5] Wang W, Fan J, Huang G et al Prevalence of kidney stones in mainland China: A systematic review. Scientific Reports, 2017, 7:41630. doi:10.1038/srep41630.

[6] Scales CD Jr, Smith AC, Hanley JM, Saigal CS Prevalence of kidney stones in the United States, EurUrol, 2012, 62(1):160-5. doi: 10.1016/j.eururo.2012.03.052.

[7] Gross AJ, Knipper S, Netsch C. Managing caliceal stones. Indian Journal of Urology: IJU: Journal of the Urological Society of India. 2014;30(1):92-98. doi:10.4103/09701591.124214.

[8] Cho SY, Na W, Lee SW, et al. Medical expulsive therapy for ureter stone using naftopidil: A multicenter, randomized, doubleblind, and placebo-controlled trial. Schwentner C, ed. PLoS ONE. 2017;12(4): e0174962. doi: 10.1371/journal.pone.0174962.

[9] Srisubat A, Potisat S, Lojanapiwat B, Setthawong V, Laopaiboon M Extracorporeal shock wave lithotripsy (ESWL) versus percutaneous nephrolithotomy (PCNL) or retrograde intrarenal surgery (RIRS) for kidney stones. Cochrane Database of Systematic Reviews, 2014, Issue 11. Art. No.: CD007044. DOI: 10.1002/14651858.CD007044.pub3.

[10] Chaussy C, Schmiedt E Extracorporeal shock wave lithotripsy (ESWL) for kidney stones. An alternative to surgery? UrolRadiol, 1984, 6: 80. doi:10.1007/BF02923707.

[11] Skolarikos A, Alivizatos G, De la Rosette J "Extracorporeal shock wave lithotripsy 25 years later: complications and their prevention," European Urology, 2006, 50, 5, 981-990.

[12] White WM, Morris SA, Klein FA, Waters WB Splenic rupture following shock wave lithotripsy. Can J Urol. 2008, 15:4196-4199.

[13] Marinkovic SP, Marinkovic CM, Xie D Spleen injury following left extracorporeal shockwave lithotripsy (ESWL), BMC Urology, 2015, 15:4. doi:10.1186/1471-2490-15-4.

[14] Akbulut F, Kucuktopcu O, Ucpinar B, et al A Rare Complication of Extracorporeal Shock Wave Lithotripsy: Intrarenal Hematoma Mimicking Pelvis RenalisTumor, Case Reports in Urology, 2015, Article ID 719618, 4 pages, doi:10.1155/2015/719618.

[15] Krambeck AE, Gettman MT, Rohlinger AL, Lohse CM, Patterson DE, Segura JW Diabetes Mellitus and Hypertension Associated with Shock Wave Lithotripsy of Renal and Proximal Ureteral Stones at 19 Years of Followup, J Urol, 2006, 175(5):1742-7.

[16] McAteer JA, Evan AP The Acute and LongTerm Adverse Effects of Shock Wave Lithotripsy. Seminars in nephrology, 2008, 28(2):200-213, doi: 10.1016/j.semnephrol.

[17] Keeley FX, Jr, Tilling K, Elves A, Menezes P, Wills M, Rao N, et al. Preliminary results of a randomized controlled trial of prophylactic shock wave lithotripsy for small asymptomatic renal calyceal stones. BJU Int. 2001; 87:1-8.

[18] Ludwig WW, Ziemba JB, Matlaga BR. Opinion: Do not treat. International Brazilian Journal of Urology: official journal of the Brazilian Society of Urology. 2016;42(2):185$187 . \quad$ doi: $10.1590 /$ S16775538.IBJU.2016.02.04.

[19] Türk C, Petrrík A, Sarica K, Seitz C et al. EAU Guidelines on Diagnosis and Conservative Management of Urolithiasis, EurUrol, 2016;69:468-74.

[20] Travaglini F, Bartoletti R, Gacci M, Rizzo M. Pathophysiology of Reno-Ureteral Colic, UrolInt 2004;72(suppl 1):20-23, https://doi.org/10.1159/000076586.

[21] Bagga HS, Chi T, Miller J, Stoller ML. New Insights Into the Pathogenesis of Renal 
Calculi. The Urologic clinics of North America. 2013;40(1):1-12.doi:10.1016/j.ucl.2012.09.006.

[22] Allen S. The Origin of Chronic Diseases Can Be in Capillary Pathology: An Evidence from Clinical Trials on Thermobalancing Treatment of Prostate Reveals, Achievements in the Life Sciences, 2016, 10, 2, 197-202.

[23] Rohrmann S, Katzke V, Kaaks R Prevalence and Progression of Lower Urinary Tract Symptoms in an Aging Population, Urology. 2016 Sep; 95:158-63. doi: 10.1016/j.urology.2016.06.021.

[24] LukacsB, CornuJN, AoutM et al Management of Lower Urinary Tract Symptoms Related to Benign Prostatic Hyperplasia in Real-life Practice in France: A Comprehensive Population Study, Eur Urol. 2013 Sep;64(3):493-501.

[25] Corona G, Vignozzi L, Rastrelli G, Lotti F, Cipriani S, Maggi M. Benign Prostatic Hyperplasia: A New Metabolic Disease of the Aging Male and Its Correlation with Sexual Dysfunctions. Int J Endocrinol. 2014; 2014:329456.

[26] Yun-kuan Y, You-guang A, You-ping HJ, Observations on the treatment of 37 cases of senile prostate hyperplasia with acupuncture,Acupunct. Tuina. Sci. 2005, 3: 17. https://doi.org/10.1007/BF02851658.

[27] Wang Y, Liu BY, Yu JN, Wu JN, Liu ZS. Electroacupuncture for moderate and severe benign prostatic hyperplasia: a randomized controlled trial. PLoS ONE. 2013; 8, e59449. pmid:23593139.

[28] Zhang W, Ma L, Bauer BA, Liu Z, Lu Y. Acupuncture for benign prostatic hyperplasia: A systematic review and meta-analysis. PLoS ONE. 2017, 12(4): e0174586. https://doi.org/10.1371/journal.pone.0174586

[29] Keehn A, Lowe FC Complementary and alternative medications for benign prostatic hyperplasia, Can J Urol. 2015 Oct;22 Suppl 1:18-23.

[30] Abrams P, Chapple C, Khoury S, et al. Evaluation and Treatment of Lower Urinary Tract Symptoms in Older Men, J Urol. 2009 Apr;181(4):1779-87.

[31] Prakash K, Pirozzi G, Elashoff M, Munger W, Waga I, Dhir R, Kakehi Y, Getzenberg RH, Symptomatic and asymptomatic benign prostatic hyperplasia: molecular differentiation by using microarrays, ProcNatlAcadSci U S A. 2002, May 28;99(11):7598-603.

[32] Huang DY, Wu JH, Sun ZY. Categories and characteristics of BPH drug evaluation models: a comparative study. Zhonghua Nan KeXue. 2014, Feb;20(2):181-5.
[33] Allen S, Aghajanyan IG Use of thermobalancing therapy in ageing male with benign prostatic hyperplasia with a focus on etiology and pathophysiology, Aging Male, 2016,

$1-5$. http://dx.doi.org/10.1080/13685538.2016.1247 151.

[34] Magistro G, Wagenlehner FM, M. Weidner GW, Stief CG, Nickel JC Contemporary management of chronic prostatitis/chronic pelvic pain syndrome,European Urology, 2016, vol. 69, no. 4, 286-297.

[35] Krieger JN, Riley DE, Cheah PY, Liong ML, Yuen KH. Epidemiology of prostatitis: new evidence for a world-wide problem, World J Urol. 2003 Jun;21(2):70-4.

[36] Cohen D, Gonzalez J, Goldstein I The Role of Pelvic Floor Muscles in Male Sexual Dysfunction and Pelvic Pain, Sex Med Rev 2016:4; 53e62. Vellucci R. Heterogeneity of chronic pain, Clin Drug Investig. 2012 Feb, 22;32 Suppl 1:3-10. doi: 10.2165/11630030000000000-00000. Kessler. TM Chronic pelvic pain syndrome: Light at the end of the tunnel? European Urology, 2016, 69 - 2, 298-299.

[37] Cho IC, Min SK "Proposed new pathophysiology of chronic prostatitis/chronic pelvic pain syndrome," Urogenital Tract Infection, 2015, 10-2, 92-101.

[38] McBride JA, Carson CC, Coward RM Testosterone deficiency in the aging male, TherAdvUrol 2016 Feb;8(1):47-60.

[39] Hackett G An update on the role of testosterone replacement therapy in the management of hypogonadism, TherAdvUrol 2016 Apr 9;8(2):147-60.

[40] Pang R, Lu J, Zhou X, Gao X, Zhang Y MP2502 testosterone replacement therapy for hypogonadal patients with chronic prostatitis/chronic pelvic pain syndrome, J Urol. 2015, 193-4, Suppl., e285, doi: http://dx.doi.org/10.1016/j.juro.2015.02.12 05

[41] Gurel B, Lucia MS, Thompson IM et al., Chronic inflammation in benign prostate tissue is associated with high-grade prostate cancer in the placebo arm of the prostate cancer prevention trial, Cancer Epidemiological BiomarkersPreview,2014, vol. 5, 847.

[42] Aghajanyan, I.G.; Allen, S. Positive Response to Thermobalancing Therapy Enabled by Therapeutic Device in Men with NonMalignant Prostate Diseases: BPH and Chronic Prostatitis. Diseases 2016, 4, doi:10.3390/diseases4020018.

[43] Allen S, Aghajanyan IG Efficacy of Thermobalancing therapy for chronic prostatitis /chronic pelvic pain syndrome, confirmed by 
clinical study, may suggest ethology and pathophysiology of this disease, Can UrolAssoc J, 2017 Nov 11. doi: 10.5489/cuaj.4473.

[44] Chung SD, Lin HC. Association between chronic prostatitis/chronic pelvic pain syndrome and anxiety disorder: a populationbased study, PLoS One 2013; 8: e64630.

[45] Krsmanovic A, Tripp DA, Nickel JC, et al. Psychosocial mechanisms of the pain and quality of life relationship for chronic prostatitis/chronic pelvic pain syndrome (CP/CPPS). Can UrolAssoc J 2014; 8: 403408.

[46] Martin S, Vincent A, Taylor AW et al Lower Urinary Tract Symptoms, Depression, Anxiety and Systemic Inflammatory Factors in Men: A Population-Based Cohort Study, PLoS One. 2015 Oct 7;10(10):e0137903

[47] Pinto JD, He HG, Chan S.W, Wang W Healthrelated quality of life and psychological wellbeing in men with benign prostatic hyperplasia: An integrative review. Jpn. J. Nurs. Sci. 2016, doi:10.1111/jjns.12115.

[48] Baldwin, A.L. A brief history of capillaries and some examples of their apparently strange behaviour. Clin. Exp. Pharmacol. Physiol. 2000, 27, 821-825.

[49] Hansen-Smith, F.M. Capillary network patterning during angiogenesis. Clin. Exp. Pharmacol. Physiol. 2000, 27, 830-835.

[50] Allen, S.; Adjani, A. Therapeutic Device and Method, United States Patent and Trademark Office. U.S. Patent 9,408,744 B2, 9 August 2016. Available online: https://www.google.com/patents/US9408744 (accessed on 22 November 2017).

[51] Shang W, Li L, Ren Y, et al. History of kidney stones and risk of chronic kidney disease: a meta-analysis. Knight J, ed. PeerJ. 2017;5:e2907. doi:10.7717/peerj.2907.

[52] El-Zoghby ZM1, Lieske JC, Foley RN, Bergstralh EJ, Li X, Melton LJ 3rd, Krambeck AE, Rule AD, Urolithiasis and the risk of ESRD, Clin J Am SocNephrol. 2012 Sep;7(9):1409-15.

[53] Wood K, Keys T, Mufarrij P, Assimos DG. Impact of Stone Removal on Renal Function: A Review. Reviews in Urology. 2011;13(2):73-89.

[54] Dai M, Zhao A, Liu A, You L, Wang P., Peiyu Wang, . Factors and Risk of Kidney Stone: A Case-Control Study in Southern China, J RenNutr. 2013 Mar;23(2):e21-8. doi: 10.1053/j.jrn.2012.04.003.

[55] Wong Y, Cook P, Roderick P, Somani BK, Metabolic Syndrome and Kidney Stone Disease: A Systematic Review of Literature, $\underline{\mathbf{J}}$ Endourol. 2016 Mar;30(3):246-53. doi: 10.1089/end.2015.0567.
[56] Han H, Segal AM, Seifter JL, Dwyer JT. Nutritional Management of Kidney Stones (Nephrolithiasis). Clinical Nutrition Research. 2015, 4(3): 137-152. doi:10.7762/cnr.2015.4.3.137.

[57] Fourcade RO, Lacoin F, Rouprêt M, et al. Outcomes and general health-related quality of life among patients medically treated in general daily practice for lower urinary tract symptoms due to benign prostatic hyperplasia. World $\mathrm{J}$ Urol. 2012; 30:419-26.

[58] Traish A, Haider KS, Doros G, Haider A. Long-term dutasteride therapy in men with benign prostatic hyperplasia alters glucose and lipid profiles and increases severity of erectile dysfunction, HormMolBiolClinInvestig. 2017, Jun 21;30(3). doi: https://doi.org/ 10.1515/hmbci-2017-0015

[59] Naeem B, Giorgio G, Akshay S et al Morbidity and Mortality After Benign Prostatic Hyperplasia Surgery: Data from the American College of Surgeons National Surgical Quality Improvement Program, Journal of Endourology, 2014, 28(7): 831-840.

[60] Elshal AM, Elmansy HM, Elkoushy MA, Elhilali MM. Male sexual function outcome after three laser prostate surgical techniques: a single center perspective, Urology, 2012, 80:1098-104.

[61] Brown CT, Emberton M, Self-management for men with lower urinary tract symptoms Curr prostate rep, 2009, 7: 111-116. https://doi.org/ 10.1007/s11918-009-0016-8.

[62] Allen S, Aghajanyan IG. Thermobalancing conservative treatment for moderate-to-lowdegree lower urinary tract symptoms (LUTS) secondary to prostate enlargement, Cogent Medicine, 2016, 3(1), 1195067.

[63] Cohen JM, Fagin AP, Hariton E, Niska JR, Pierce MW, et al. Therapeutic Intervention for Chronic Prostatitis/Chronic Pelvic Pain Syndrome (CP/CPPS): A Systematic Review and Meta-Analysis. PLOS ONE 2012 7(8): e41941. https://doi.org/10.1371/journal.pone.00 41941

[64] Krsmanovic A, Tripp DA, Nickel JC, et al. Psychosocial mechanisms of the pain and quality of life relationship for chronic prostatitis/chronic pelvic pain syndrome (CP/CPPS). Canadian Urological Association Journal. 2014;8(11-12):403-408. doi:10.5489/cuaj.2179

[65] Cheng S, Shi B, Zhu Y, et al. AB058. Therapeutic efficacy of trans-rectal triple physiotherapy combined with pharmacotherapy in comparison with pharmacotherapy alone in patients with chronic prostatitis/chronic pelvic pain syndrome (CP/CPPS). Translational 
Treatment of Kidney Stone Disease, Benign Prostatic Hyperplasia and Chronic Prostatitis with Thermobalancing Therapy should be listed in the Guidance for Health Professionals

Andrology and Urology. 2017;6(Suppl 3): AB058. doi:10.21037/tau. 2017.s058.

[66] Rees, J., Abrahams, M., Doble, A., Cooper, A. and the Prostatitis Expert Reference Group (PERG). Diagnosis and treatment of chronic bacterial prostatitis and chronic prostatitis/chronic pelvic pain syndrome: a consensus guideline. BJU Int, 2015 ,116: 509525. doi:10.1111/bju.13101

[67] Kucuk EV, Suceken FY, Bindayi A, et al. Effectiveness of acupuncture on chronic prostatitis-chronic pelvic pain syndrome category IIIB patients: a prospective, randomized, nonblinded, clinical trial. Urology 2015; 85:636-40

[68] Zhou M, Yang M, Chen L, Yu C, Zhang W, Ji $\mathrm{J}$, et al. The effectiveness of long-needle acupuncture at acupoints BL30 and BL35 for
CP/CPPS: a randomized controlled pilot study. BMC Complement Altern Med. 2017;17(1):263. doi: 10.1186/s12906-0171768-2.

[69] Chang SC, Hsu CH, Hsu CK, Yang SSD, Chang SJ. The efficacy of acupuncture in managing patients with chronic prostatitis/chronic pelvic pain syndrome: A systemic review and meta-analysis, NeurourolUrodyn. 2017 Feb;36(2):474-481. doi: $10.1002 /$ nau. 22958 .

[70] Allen S, Aghajanyan IG New independent thermobalancing treatment with therapeutic device for internal diseases: chronic prostatitis and chronic pelvic pain syndrome, Nephrourol Mon, 2017, e44694. doi: "http://dx.doi.org/10.5812/numonthly.44694"10 $.5812 /$ numonthly.44694.

Citation: Simon Allen, Treatment of Kidney Stone Disease, Benign Prostatic Hyperplasia and Chronic Prostatitis with Thermobalancing Therapy Should Be Listed in The Guidance for Health Professionals.ARC Journalof Urology.2018; 3(2):1-11. doi:dx.doi.org/10.20431/2456-060X.0302001.

Copyright:(C) 2018 Authors. This is an open-access article distributed under the terms of the Creative Commons Attribution License, which permits unrestricted use, distribution, and reproduction in any medium, provided the original author and source are credited. 